\title{
Cognitive functioning in schizophrenia, schizoaffective disorder and affective psychoses: meta-analytic study
}

Emre Bora, Murat Yucel and Christos Pantelis

\section{Background}

Cognitive functioning in affective psychosis and schizoaffective disorder is much less studied compared with schizophrenia.

\begin{abstract}
Aims
To quantitatively undertake a meta-analysis of the available data that directly compares cognitive functioning across schizophrenia, schizoaffective disorder and affective psychosis.

\section{Method}

Following a thorough literature review, 31 studies that compared the performances of people with schizophrenia (1979 participants) with that of those with affective psychosis or schizoaffective disorder (1314 participants) were included. To determine the effect of demographic and clinical confounders, meta-regression and subgroup analyses were conducted.
\end{abstract}

\section{Results}

In 6 of 12 cognitive domains, people with schizophrenia performed worse than people with schizoaffective disorder or affective psychosis. However, the between-group differences were small and the distribution of effect sizes showed substantial heterogeneity. The between-group differences were driven by a higher percentage of males, more severe negative symptoms and younger age at onset of illness in the schizophrenia samples.

\section{Conclusions}

Neuropsychological data do not provide evidence for categorical differences between schizophrenia and other groups. However, a subgroup of individuals with schizophrenia who have more severe negative symptoms may be cognitively more impaired than those with affective psychosis/schizoaffective disorder.

\section{Declaration of interest}

None.
Recent findings from genetic studies have revitalised the debate about the validity of Kraepelin's classification of the major psychoses. Although the Krapelenian notion would predict separate susceptibility genes for bipolar disorder and schizophrenia, there is increasing evidence for common genetic susceptibility factors for both disorders. Interestingly, suggested genetic susceptibility factors like neuregulin or disrupted in schizophrenia (DISC1) seem to be more related with the clinical entities that lie in an intermediate position between the schizophrenia-bipolar disorder continuum (such as psychotic bipolar disorder and schizoaffective disorder). ${ }^{1}$ The individuals that present with a mixture of psychotic and affective features have always caused discussion about classification of the major psychoses. Focusing on these intermediate entities that have been labelled with different names, such as mixed psychoses or schizoaffective spectrum, could help us to delineate the boundaries between the major psychoses and improve our classification systems. ${ }^{2-4}$

Neuropsychological studies have also provided evidence for cognitive impairment in similar cognitive domains in schizophrenia and bipolar disorder. Several recent meta-analyses support the presence of cognitive impairments in individuals with euthymic bipolar disorder, especially deficits in executive functions, verbal memory and attention. ${ }^{5-7}$ However, a meta-analytic study that compared the cognitive profiles of people with schizophrenia and people with bipolar disorder provided evidence for more severe impairments in schizophrenia. ${ }^{8}$ Medium effect-size differences in cognitive domains like executive function, memory and mental speed seemed to separate schizophrenia from bipolar disorder. Studying cognitive functions in mixed psychoses may help us to explain these conflicting findings. Further, symptom differences between these disorders may be relevant, for example, negative symptoms have been particularly linked to executive function deficits in the disorder. ${ }^{9}$ So far, relatively few studies have investigated cognitive functioning in schizoaffective disorder and affective psychoses. To our knowledge this is the first quantitative review comparing neuropsychological functioning in mixed psychoses and schizophrenia.

Our aim was to review the studies that compared cognitive functioning in people with schizophrenia, schizoaffective disorder and mood disorders with psychotic features, and to examine the influence of clinical and demographic variables on possible group differences.

\section{Method}

\section{Study selection}

Potential articles were identified thorough a literature search in PubMed, Scopus, PsychINFO, EMBASE and Medline in the period between January 1975 and December 2007. For the literature search, combinations of the following keywords were used: schizophrenia, bipolar disorder, manic-depress ${ }^{\star}$, mania, depress ${ }^{\star}$, schizoaff*, affective psychosis, cognit ${ }^{\star}$, neuropsycholog* ${ }^{\star}$. The last two terms were also replaced with the keywords describing cognitive domains (attention, executive, memory). The reference lists of the published articles were also reviewed.

The inclusion criteria for the meta-analysis were as follows.

(a) The study assessed cognitive abilities using reliable neuropsychological testing methods and were published in English language peer-reviewed journals.

(b) The study compared adults with schizophrenia and people with schizoaffective disorder and/or affective psychosis (psychotic depression, psychotic bipolar disorder/mania) (according to research diagnostic criteria, ICD-9, ICD-10, DSM-III, DSM-III-R or DSM-V). 
(c) The study reported test scores (means and standard deviations) of both groups or effect sizes of group comparisons.

(d) The study incluedd a cognitive task that was reported in at least three other studies.

(e) The results reported separately for each task (not as composite scores).

(f) The results reported independent data. If there were several relevant articles based on a single sample, the study with the larger sample was selected.

Recorded variables were:

(a) name of the first author and year of publication;

(b) diagnoses;

(c) sample characteristics (number of participants and percentage of males in each group) and status of individuals (in-patient/ out-patient);

(d) mean and standard deviations for demographic (age, duration of education) and clinical variables (age at onset, duration of illness, positive and negative symptoms), and dosage of antipsychotic (in chlorpromazine equivalents);

(e) results of the cognitive tests used.

In the first instance, abstracts and titles of articles were reviewed to identify relevant studies. This resulted in 163 articles that were examined further. One hundred and six of these studies compared the cognitive profile of people with schizophrenia with that of those with schizoaffective disorder, bipolar disorder or depression. Later, bipolar disorder or psychotic depression samples were examined for the presence of psychotic symptoms. Some studies that compared people with schizophrenia and schizoaffective disorder/affective psychosis were excluded as the schizoaffective disorder/affective psychosis group included individuals with other psychotic diagnoses like delusional disorder. Only 51 studies compared individuals with schizoaffective disorder or affective psychosis to those with schizophrenia. Fourteen other studies did not provide sufficient data to calculate effect sizes (or reported scores from combined individual tasks or batteries like Luria-Nebraska) and reported tasks that were used in less than four studies. The remaining 37 studies were checked for sample overlap. Six studies were excluded since they were based on the same sample used in other studies. The final selection of 31 studies included 1979 people with schizophrenia and 1314 people with schizoaffective disorder or affective psychosis (online Table DS1). ${ }^{10-40}$

\section{Neuropsychological variables}

The following neuropsychological variables were used in the studies. Mental speed: Trail Making Test Part A (TMT-A), Digit Symbol Substitution Test (DSST) and symbol digit modalities tests; ${ }^{40-42}$ Wisconsin Card Sorting Test (WCST): categories achieved or number of perserative errors was used to assess concept formation and shifting ability; ${ }^{43}$ Trail Making Test Part B: TMT-B and TMT B-A tests were used; ${ }^{41}$ verbal fluency: semantic and phonetic fluency tests; ${ }^{44}$ verbal working memory: Backwards digit span, letter-number sequencing and Working Memory Index; ${ }^{42,45}$ spatial working memory: assessed by different versions of Delayed Response Tasks; $;^{22,30,46}$ immediate verbal memory: word list from Rey Auditory Verbal Learning Test (RAVLT), California Verbal Learning Test (CVLT), word list subtest of the Battery of Memory Efficiency (BEM), Hopkins Verbal Learning Test (HVLT), Miller-Selfridge task, story recall from the Logical memory subtest of the Wechsler Memory Scale (WMS), the History subtest of BEM and Babcock story recall ${ }^{47-52}$ delayed verbal memory: Word list, delayed recall of word list (the RAVLT, CVLT, BEM) or story recall (either the Logical memory of WMS, History subtest from BEM, or Babcock story recall); immediate visual memory: Rey Osterich Complex Figure (ROCF), WMS-R Visual memory, BEM; ${ }^{49,52-53}$ delayed visual memory: delayed recall version of ROCF, Visual reproduction of WMS, BEM, Benton Visual Retention Test (BVRT); ${ }^{49,52-54}$ attention: Forwards digit span or Total digit span; ${ }^{42}$ global cognition: full Wechsler Adult Intelligence Scale IQ (WAIS) and WAIS IQ estimates. ${ }^{42}$

For the purpose of analysing the effect of confounding variables on cognition, three summed scores were calculated by averaging the effect sizes of individual tasks: individual effect sizes for verbal memory and visual memory were used to calculate the memory score; the WCST and TMT-B scores were summed to provide an executive function score. Since verbal fluency performance of people with schizophrenia has been reported to be best predicted by mental speed tasks, ${ }^{55}$ a summed score of these tasks - psychomotor speed - was also calculated.

\section{Statistical analyses}

For each cognitive test an effect size (Cohen's $d$ ) and standard error was calculated. Effect sizes were weighted using the inverse variance method. We used a random effects model (DerSimonian-Laird estimate). When multiple tests were reported for one cognitive domain, one combined effect size was calculated for these tests. Whenever participants with schizophrenia performed more poorly than those with schizoaffective disorder or affective psychosis, we reported between-group differences as positive effect sizes. Therefore, the effect sizes for the relevant variables were multiplied by minus one. Homogeneity of the resulting mean-weighted effect sizes was tested using the Q-test. Squared tau $\left(\tau^{2}\right)$ value was also used as a measure of homogeneity. We also calculated homogeneity statistics $Q_{\text {bet }}$ to test the significance of differences between schizophrenia/schizoaffective disorder and schizophrenia/affective psychosis comparisons. Publication bias was assessed by Egger's test. Meta-analyses were performed using MIX software version 1.7 on Windows. ${ }^{56}$

Meta-regression analyses were used to estimate the impact of demographic (age, gender) and clinical (age at onset, duration of illness, positive symptoms and negative symptoms) variables, and antipsychotic levels (expressed as chlorpromazine equivalents) on between-group differences. Meta-regression analyses were conducted in SPSS 11.0 using Windows by using the macros written by David B. Wilson. This procedure allowed us to perform weighted generalised least squares regression. Meta-regression analyses performed with a random effects model was performed using the restricted-information maximum likelihood method with a significance level set at $P<0.05$.

\section{Results}

Schizophrenia and schizoaffective disorder/affective psychosis studies were not well matched demographically for gender. There was a significantly higher ratio of male participants with schizophrenia (schizophrenia 71.9\%, schizoaffective disorder/affective psychosis 52.1\%; odds ratio $(\mathrm{OR})=2.14,95 \%$ CI 1.65-2.77, $z=5.75, P<0.0001)$. There were also more males with schizoaffective disorder (59\%) compared with affective psychosis (44\%). Participants with schizophrenia were younger compared with those with schizoaffective disorder/affective psychosis $(d=0.15,95 \%$ CI $0.00-0.29, z=1.97, P=0.05)$. There was also a difference in educational level, alhough with a small effect size, with those with schizophrenia being less educated compared with 
the schizoaffective disorder/affective psychosis group $(d=0.19$, $95 \% \mathrm{CI}=0.09-0.29, z=3.78, P=0.002)$. Whereas duration of illness $(d=0.02,95 \% \mathrm{CI}=-0.15$ to $0.18, z=0.19, P=0.85)$ was similar between groups, age at onset was significantly younger $(d=0.24,95 \% \mathrm{CI}=0.07-0.42, z=2.70, P=0.007)$ in the schizophrenia group.

Participants with schizophrenia performed significantly worse in 6 out of 12 tests (Table 1). There were significant differences for verbal memory (Fig.1), IQ, verbal working memory, TMT-B and WCST performances, and there was a trend for a difference on mental speed and fluency tasks. All of the significant effect sizes were small (Cohen's $d$ scores between 0.25 and 0.42). There were no significant between-group differences for visual memory, attention (digit span) or spatial working memory tests. There was a significant heterogeneity of distribution of effect sizes for 7 out of 12 domains. Squared tau values of the tasks also changed from near-zero values indicating homogenous distribution (minimum $\tau^{2}=0.003$ for WCST) to highly heterogeneous values (maximum $\tau^{2}=0.11$ for mental speed). The heterogeneity was especially substantial for TMT-B, mental speed and visual memory tasks. Two of the studies especially contributed to the heterogeneous distribution of effect sizes for TMT-B and mental speed tasks (Fig. 2). ${ }^{18,33}$ There was no publication bias apparent for any of the tasks (Table 1).

There was also a small but significant between-group difference for the summary measures (Table 2). Q-tests for one of these measures, psychomotor speed, also indicated there was significant heterogeneity.

\section{Analysis of moderator variables}

Meta-regression analyses

We conducted meta-regression analyses for age (28 studies), education (19 studies), age at onset and duration of illness (18 studies), chlorpromazine equivalents (10 studies), positive and negative symptoms (9 studies).

\begin{tabular}{|c|c|c|c|c|c|c|c|c|c|}
\hline Test & $\begin{array}{c}\text { Studies } \\
n\end{array}$ & $\begin{array}{l}\text { Schizophrenia } \\
\text { group, } \\
n\end{array}$ & $\begin{array}{c}\text { Schizoaffective/affective } \\
\text { psychosis group } \\
n\end{array}$ & $d$ & $95 \% \mathrm{Cl}$ & $z$ & $P$ & $Q$-test $P$ & Bias \\
\hline Verbal memory immediate & 8 & 749 & 364 & 0.42 & 0.20 to 0.65 & 3.67 & 0.0002 & 0.02 & 0.94 \\
\hline $\begin{array}{l}\text { Wechsler Adult Intelligence } \\
\text { Scale IQ }\end{array}$ & 7 & 499 & 205 & 0.37 & 0.09 to 0.65 & 2.61 & 0.009 & 0.03 & 0.49 \\
\hline Verbal working memory & 7 & 312 & 208 & 0.31 & 0.02 to 0.57 & 2.13 & 0.02 & 0.06 & 0.81 \\
\hline Verbal memory delayed & 9 & 727 & 345 & 0.29 & 0.09 to 0.49 & 2.80 & 0.005 & 0.07 & 0.90 \\
\hline Mental speed & 12 & 1154 & 743 & 0.26 & 0.03 to 0.49 & 2.24 & 0.02 & $<0.0001$ & 0.54 \\
\hline Wisconsin Card Sorting Test & 15 & 811 & 412 & 0.25 & 0.12 to 0.38 & 3.78 & 0.0002 & 0.39 & 0.60 \\
\hline Trail Making Test Part B & 10 & 795 & 644 & 0.23 & 0.00 to 0.47 & 1.91 & 0.06 & 0.001 & 0.30 \\
\hline Fluency & 9 & 537 & 359 & 0.22 & -0.03 to 0.48 & 1.69 & 0.09 & 0.002 & 0.09 \\
\hline Visual memory immediate & 4 & 318 & 205 & 0.14 & -0.21 to 0.50 & 0.79 & 0.43 & 0.03 & 0.05 \\
\hline Visual memory delayed & 8 & 658 & 332 & 0.09 & -0.24 to 0.40 & 0.48 & 0.63 & $<0.0001$ & 0.19 \\
\hline Digit span & 12 & 924 & 352 & 0.02 & -0.14 to 0.18 & 0.28 & 0.78 & 0.17 & 0.95 \\
\hline Spatial working memory & 4 & 87 & 77 & -0.09 & -0.55 to 0.38 & 0.37 & 0.71 & 0.09 & 0.90 \\
\hline
\end{tabular}

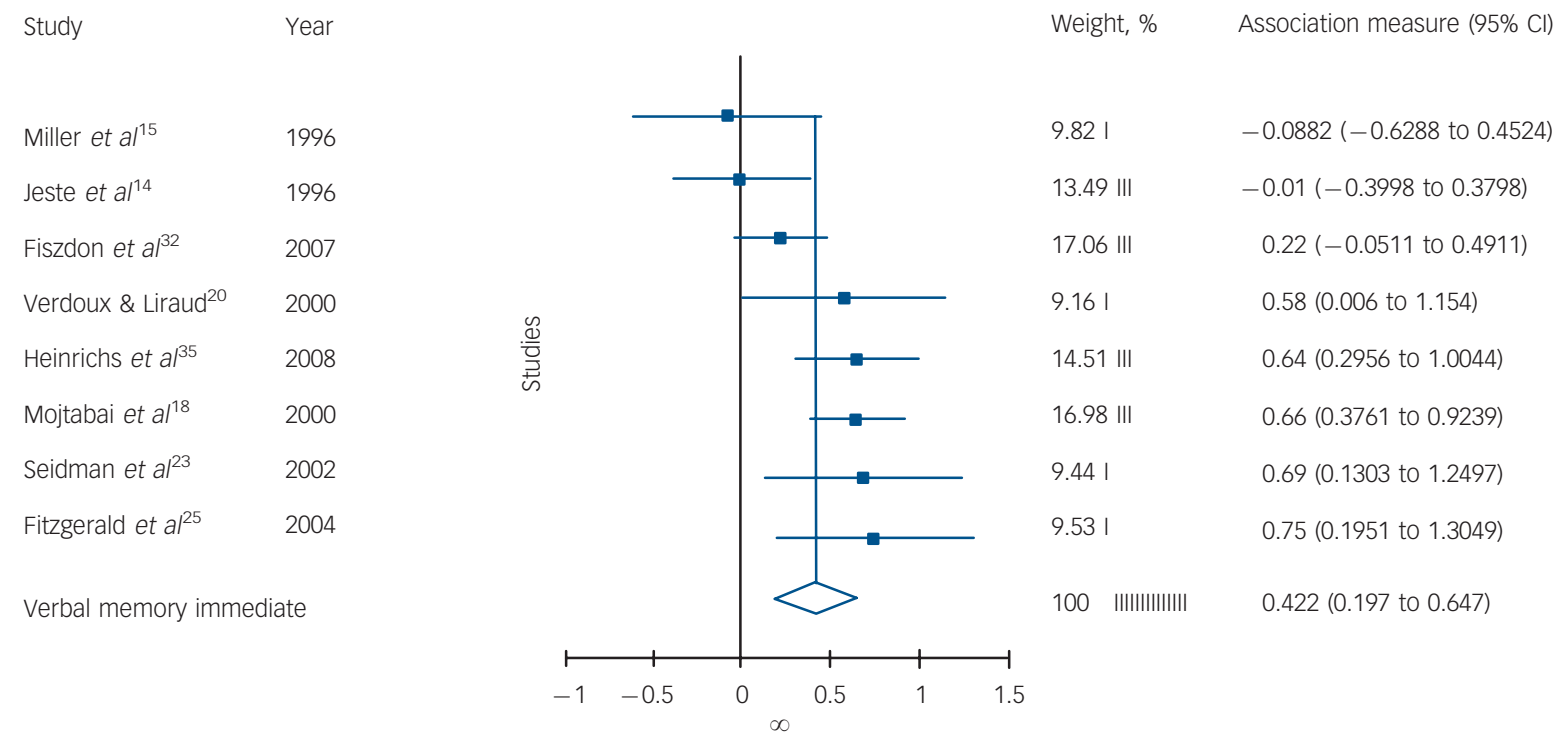




\begin{tabular}{|c|c|c|c|c|c|c|}
\hline Test & Studies, $n$ & $d$ & $95 \% \mathrm{Cl}$ & $z$ & $P$ & $Q$-test $P$ \\
\hline Executive function & 19 & 0.23 & 0.08 to 0.38 & 2.94 & 0.003 & 0.002 \\
\hline Affective psychosis & 12 & 0.28 & 0.11 to 0.46 & 3.13 & 0.002 & 0.04 \\
\hline Schizoaffective disorder & 9 & 0.12 & -0.06 to 0.31 & 1.30 & 0.19 & 0.11 \\
\hline Matched for gender & 9 & 0.08 & -0.06 to 0.23 & 1.18 & 0.24 & 0.28 \\
\hline Stable participants & 13 & 0.22 & 0.04 to 0.40 & 2.41 & 0.02 & 0.001 \\
\hline Memory & 13 & 0.27 & 0.11 to 0.43 & 3.37 & 0.001 & 0.12 \\
\hline Affective psychosis & 7 & 0.30 & 0.05 to 0.55 & 2.39 & 0.02 & 0.07 \\
\hline Schizoaffective disorder & 6 & 0.23 & 0.04 to 0.43 & 2.32 & 0.02 & 0.35 \\
\hline Matched for gender & 6 & 0.08 & -0.16 to 0.32 & 0.63 & 0.53 & 0.13 \\
\hline Stable participants & 6 & 0.31 & 0.09 to 0.52 & 2.80 & 0.005 & 0.10 \\
\hline Psychomotor speed & 17 & 0.24 & 0.07 to 0.42 & 2.79 & 0.005 & 0.001 \\
\hline Affective psychosis & 11 & 0.27 & 0.03 to 0.51 & 2.16 & 0.03 & 0.001 \\
\hline Schizoaffective disorder & 8 & 0.22 & 0.02 to 0.43 & 2.18 & 0.03 & 0.05 \\
\hline Matched for gender & 8 & 0.14 & -0.08 to 0.35 & 1.22 & 0.22 & 0.01 \\
\hline Stable participants & 11 & 0.31 & 0.12 to 0.51 & 3.19 & 0.001 & 0.008 \\
\hline
\end{tabular}

Meta-regression analyses demonstrated an association between more significant between-group differences for negative symptoms (schizophrenia > schizoaffective disorder/affective psychosis) and more severe impairments for memory $(B=0.23$, s.e. $=10, z=2.27, P=0.02, k=5)$, psychomotor speed $(B=0.39$, s.e. $=0.09, \quad z=4.49, \quad P<0.001, k=6)$, and executive function $(B=0.41$, s.e. $=0.09, z=4.64, P<0.001, k=6)$ in people with schizophrenia compared with those with schizoaffective disorder/ affective psychosis. It means that more severe differences in these domains in schizophrenia were associated with greater severity of negative symptoms in this group. Younger age at onset of illness in the schizophrenia group (compared with schizoaffective disorder/ affective psychosis) was associated with more pronounced psychomotor impairment $(B=0.17$, s.e. $=0.19, z=1.92, P=0.05, k=10)$. In the schizophrenia and schizoaffective disorder/affective psychosis comparison, there was a significant relationship between an increased percentage of males and severity of negative symptoms in the schizophrenia groups $(B=1.05, \quad$ s.e. $=0.53, \quad z=2.0$, $P=0.04)$. The increased male ratio in the schizophrenia group was also associated with younger age at onset of illness ( $B=0.39$, s.e. $=0.20, z=1.93, P=0.05)$.

More severe positive symptoms in the schizophrenia group were also associated with more pronounced psychomotor impairment $(B=0.59$, s.e. $=0.29, z=2.08, P=0.04, k=10)$ in this group compared with other psychoses. Differences regarding matching of education also contributed to heterogeneity. In the studies in which the schizophrenia group have fewer years of education than other psychoses, there were more between-group differences for psychomotor $(B=0.69$, s.e. $=0.32, z=2.17$, $P=0.03)$ and executive $(B=0.89$, s.e. $=0.30, z=2.97, P=0.003$, $k=11)$ abilities.

Meta-regression analyses did not reveal any relationship between the other variables (age, duration of illness, chlorpromazine equivalents) and cognitive tests.

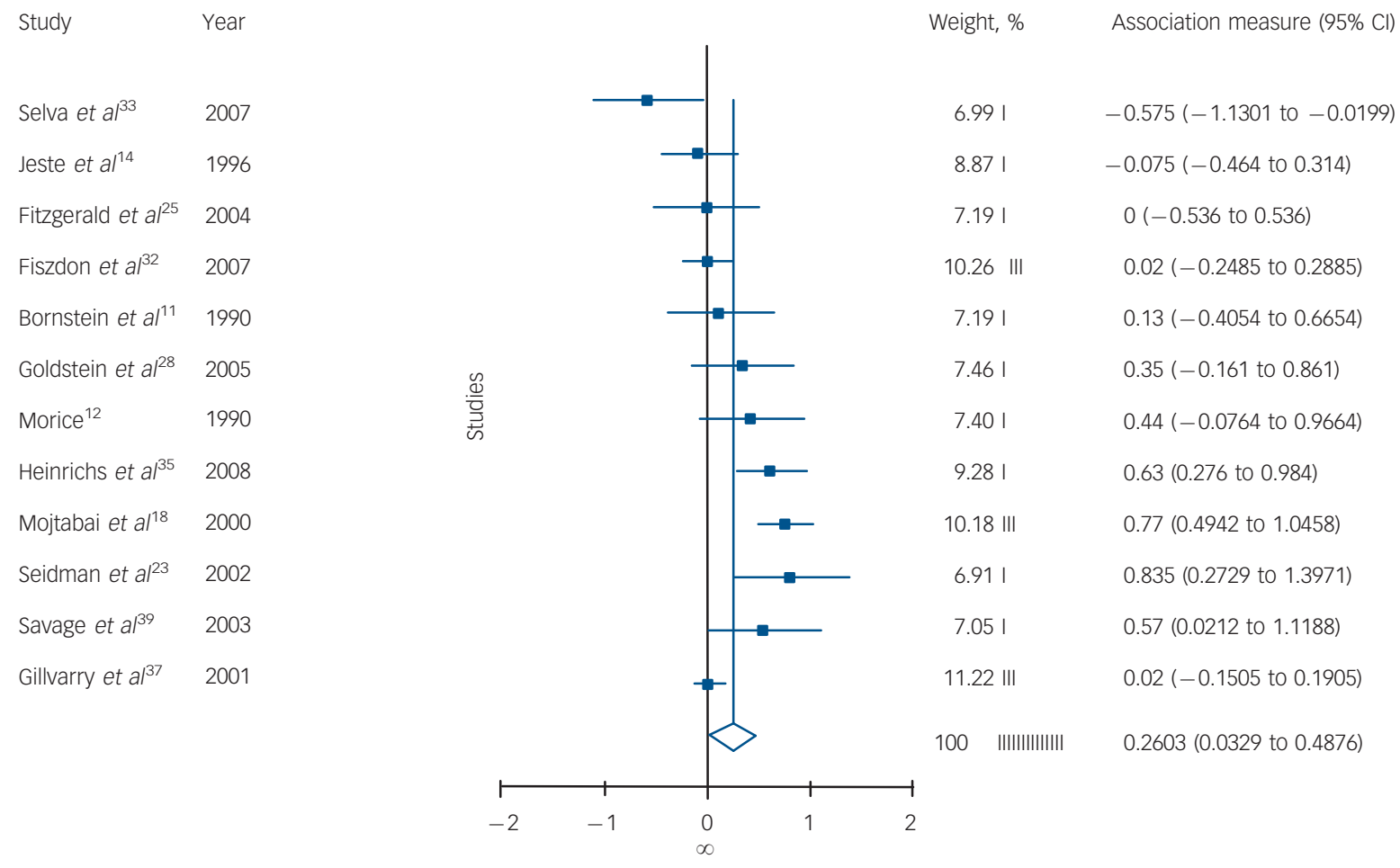


Effects of gender composition and state of illness on group differences

In addition to meta-regression analyses, further analyses were conducted to examine the impact of gender and state on group differences (Table 2). Differences between gender compositions of groups had a significant effect on results. Between-group differences for cognitive variables did not change when the analysis was restricted to studies that compared clinically stable samples.

For the analysis of the effect of gender, 14 studies in which there was an extreme male/female ratio in the schizophrenia group compared with the schizoaffective disorder/affective psychosis group were excluded. There were 15 studies remaining for this analysis. Effect sizes for between-group differences were significantly reduced in gender-matched comparisons for all summary measures. Cohen's $d$ scores for these measures $(0.08-0.15)$ were small and insignificant. Between-study heterogeneity was also reduced. The non-significant $Q$-test showed that the distribution of effect sizes for executive function and memory measures were homogeneous. Squared tau values also showed a very significant decrease in heterogeneity of executive function $\left(\tau^{2}\right.$ decreased from 0.054 to 0.009 ) and memory ( $\tau^{2}$ decreased from 0.026 to 0.002 ). These results suggest that most of the heterogeneity for memory and executive function was caused by the studies that were not matched for gender. Heterogeneity of the effect sizes for psychomotor speed tasks was not totally explainable only by gender differences. The study of Gilvarry et $\mathrm{al}^{21}$ that had a schizophrenia group with a very low premorbid IQ compared with the affective group (Cohen's $d=1.14$ ) also contributed to this hetereogenity (0.21). The $Q$-value showed that the distribution of effect sizes was sufficiently homogeneous when the study of Gilvarry et al was excluded.

\section{Impact of diagnosis}

The meta-analyses were repeated for separate schizophrenia $v$. schizoaffective disorder and schizophrenia $v$. affective psychosis comparisons (Tables 2 and 3). For executive functions and verbal memory, the magnitude of differences between schizophrenia and affective psychosis were marginally larger than differences between schizophrenia and schizoaffective disorder. However, $Q_{\text {bet }}$ test was not significant for these differences between schizophrenia/ affective psychosis and schizophrenia/schizoaffective comparisons
( $P>0.05$ for all cognitive measures). The $Q$-test also indicated that the distribution of effect sizes were homogeneous for schizoaffective disorder but not for affective psychosis.

\section{Discussion}

To our knowledge, this is the first meta-analytic study that compares cognitive performances of people with schizoaffective disorder and people with affective psychosis with those of people with schizophrenia. In a number of cognitive domains, people with schizophrenia performed more poorly than people with schizoaffective disorder/affective psychosis. However, the effect sizes of these differences were minimal. Moreover, a significant amount of the difference between the groups and nonhomogenous distribution of effect sizes were explainable by those studies that included a higher percentage of male participants in the schizophrenia groups compared with the schizoaffective disorder/affective psychosis group. Interestingly, inclusion of participants with more negative symptoms and younger age at onset of illness in the schizophrenia samples also increased the between-group differences.

A previous quantitative review examined the differences in cognitive functions between people with schizophrenia and those with bipolar disorder. ${ }^{8}$ Krabbendam et al reported relatively more severe impairment in the schizophrenia group compared with our study. The authors reported between-group differences with moderate effect sizes for verbal fluency $(d=0.63)$, verbal working memory $(d=0.60)$, executive control $(d=0.55$, a combined measure of TMT-B and Stoop test), delayed recall and mental speed $(d=0.50)$. In our study, effect sizes for these measures were only half of these values. Although Krabbendam et $a l^{8}$ also reported significant between-group differences for visual memory $(d=0.51)$, in the current study the between-group difference for this measure was negligible. However, the between-group differences for IQ and verbal memory measures were comparable with our findings.

According to our findings, diagnoses in the schizoaffective disorder/affective psychosis group only slightly affected the degree of cognitive impairment in this group relative to schizophrenia. In comparison with participants with schizophrenia, individuals with affective psychosis had a slightly better performance on verbal memory and set shifting tasks than those with schizoaffective

\begin{tabular}{|c|c|c|c|c|c|c|}
\hline Test & Studies, $n$ & $d$ & $95 \% \mathrm{Cl}$ & $z$ & $P$ & $Q$-test $P$ \\
\hline \multicolumn{7}{|l|}{ Fluency } \\
\hline Affective psychosis & 6 & 0.29 & -0.01 to 0.59 & 1.90 & 0.06 & 0.01 \\
\hline Schizoaffective disorder & 5 & 0.32 & 0.00 to 0.64 & 1.95 & 0.05 & 0.15 \\
\hline \multicolumn{7}{|l|}{ Verbal memory } \\
\hline Affective psychosis & 6 & 0.36 & 0.03 to 0.69 & 2.14 & 0.03 & 0.001 \\
\hline Schizoaffective disorder & 4 & 0.23 & 0.02 to 0.44 & 2.20 & 0.03 & 0.58 \\
\hline \multicolumn{7}{|l|}{ Trail Making Test Part B } \\
\hline Affective psychosis & 8 & 0.27 & 0.01 to 0.52 & 2.07 & 0.04 & 0.009 \\
\hline Schizoaffective disorder & 5 & 0.17 & -0.15 to 0.49 & 1.03 & 0.30 & 0.24 \\
\hline \multicolumn{7}{|l|}{ Wisconsin Card Sorting Test } \\
\hline Affective psychosis & 9 & 0.30 & 0.10 to 0.50 & 2.91 & 0.004 & 0.20 \\
\hline Schizoaffective disorder & 7 & 0.21 & 0.03 to 0.39 & 2.34 & 0.02 & 0.57 \\
\hline \multicolumn{7}{|l|}{ Mental speed } \\
\hline Affective psychosis & 8 & 0.26 & -0.10 to 0.61 & 1.43 & 0.15 & $<0.0001$ \\
\hline Schizoaffective disorder & 5 & 0.24 & 0.01 to 0.47 & 2.04 & 0.04 & 0.02 \\
\hline \multicolumn{7}{|l|}{ Visual memory } \\
\hline Affective psychosis & 5 & 0.10 & -0.27 to 0.46 & 0.53 & 0.60 & 0.01 \\
\hline Schizoaffective disorder & 4 & 0.08 & -0.35 to 0.51 & 0.35 & 0.72 & 0.02 \\
\hline
\end{tabular}


disorder. However, these differences were not statistically significant. Since there were only a few studies that directly compared the cognitive performance of schizoaffective disorder and affective psychosis, we could not perform a meta-analysis for this comparison. However, separate comparisons of schizoaffective disorder and affective psychosis with schizophrenia do not support a substantial difference on cognitive performance between schizoaffective disorder and affective psychosis groups. There are only a limited number of studies that compared the cognitive profiles of individuals with psychotic depression with those with psychotic bipolar disorder, and although available data do not suggest differences, there is a need for further studies. ${ }^{18}$

One of the moderator variables, gender, greatly contributed to observed small between-group differences and heterogeneity in cognitive functioning. This result is consistent with previous studies that reported more pronounced cognitive dysfunction in male participants with schizophrenia. ${ }^{57} \mathrm{~A}$ preliminary metaregression analysis also provided evidence for the role of negative symptoms. These results were interrelated since the studies that included a higher percentage of males in their schizophrenia groups reported more severe negative symptoms compared with schizoaffective disorder/affective psychosis groups. Between-study heterogeneity of participants with schizophrenia seems to be closely associated with outcome in various studies. The studies that include a higher ratio of males (and younger age at onset of illness) and/or individuals with severe negative symptoms in the schizophrenia group compared with the schizoaffective disorder/affective psychosis group seem to be largely responsible for significant reported differences between the two groups. Previous studies reported a higher ratio of males among the participants with more severe negative symptoms and those with deficit schizophrenia. ${ }^{57,58}$ A recent meta-analytic study examined the cognitive differences between deficit and non-deficit schizophrenia. ${ }^{59}$ These authors reported that individuals with deficit schizophrenia were more impaired compared with those with non-deficit schizophrenia. Most of the between-group differences had moderate effect sizes. According to these findings, it is reasonable to predict that cognitive performances of people with non-deficit schizophrenia would not differ significantly from those of individuals with schizoaffective disorder/affective psychosis.

Mixed affective and psychotic features are also part of the clinical presentation of some people with schizophrenia. Depression and manic features are commonly observed in individuals with schizophrenia. ${ }^{60}$ It is also interesting that affective symptoms are observed in non-deficit schizophrenia rather than deficit schizophrenia. ${ }^{61}$ Regardless of the diagnosis, individuals with mixed psychotic and mood features can have similar cognitive deficits that are less pronounced compared with those of people with schizophrenia with severe negative symptoms. These findings are compatible with recent genetic findings, which demonstrate that susceptibility factors such as Neuregulin 1 may increase the genetic risk for schizophrenia and mood disorders presenting with a mixture of psychotic and mood symptoms rather than more prototypical forms of major psychoses. ${ }^{1}$

Another important issue in classification of schizophrenia and mood disorders is the effect of psychotic features on cognitive functioning of people with mood disorder. This is also relevant to explain the differences between our meta-analysis and a previous study by Krabbendam et $a l^{8}$. Since we did not include non-psychotic bipolar disorder and depression in our meta-analysis, the effect of psychosis could explain the differences between studies. However, this is only an indirect interpretation: our study does not provide any direct evidence for the effect of psychosis in mood disorders. Studies that directly study the impact of psychosis on cognitive functioning in mood disorders are crucial to better understand cognitive impairment in mood disorders. There is already significant evidence for a negative impact of psychosis on cognitive functioning in depression and the outcome of several recent studies also showed a more significant cognitive impairment in psychotic bipolar disorder. ${ }^{62-64}$

Overall, the current findings do not support the classical Kraepelinian distinction between schizophrenia and bipolar disorder. The results may be compatible with the two different alternatives of the Kraepelinian dichotomy. First, the most severe schizophrenia and psychotic bipolar disorder may lie on the opposite ends of a continuum, as suggested by Crow and others. ${ }^{65-66}$ In that case, there should be only a quantitative change in the degree of cognitive dysfunction along the continuum from schizophrenia and psychotic mood disorders. Our results seemed to be compatible with this notion. However, results of our analysis can also suggest a second alternative, a revised version of the dichotomy. It may be the case that only people with schizophrenia with more severe negative symptoms are more impaired in certain domains. These findings related to 'deficit' schizophrenia, taken together with recent studies suggesting there may be separate genetic risk factors for schizophrenia with severe cognitive impairment, provide evidence for an alternative to the continuum model. ${ }^{67}$ Thus, it is possible that there may be a categorical distinction between a subgroup of individuals with poor outcome schizophrenia and other psychotic disorders including people with schizophrenia with a good prognosis. This possibility would suggest a categorical distinction more distal than proposed by the classical dichotomy. The jury is still out on this subject and future work that takes the subtype approach into consideration will be important in order to elucidate the nature of the major psychoses.

Results of this study might also be quite relevant regarding discussions about classification of bipolar disorder and schizophrenia in DSM-V. Recently, Keefe \& Fenton suggested that cognitive impairment should be included as an inclusion criteria for diagnosis of schizophrenia. ${ }^{68}$ One of their main arguments is the utility of cognitive impairment to increase the point of rarity between schizophrenia and bipolar disorder. Although we agree that inclusion of cognitive impairment could be desirable for some other reasons (i.e. increasing attention given to cognitive impairment in clinical practice), our results do not support the notion that cognitive impairment could help to distinguish bipolar disorder and schizophrenia.

\section{Limitations}

The current meta-analysis has some limitations relating to the availability of uniform data in the published studies. First, there were few studies that reported cognitive performances of individuals in well-defined remission. This may be important because the state of the illness may be an important confounder for cognitive functions and may have a differential effect on schizophrenia and mood disorders. We attempted to minimise the impact of state on cognitive functions by accepting being an out-patient or being close to discharge as a measure of stability of mental state, although this is not optimal. Second, only a subgroup of studies reported data regarding variables like age at onset of illness, duration of illness and symptoms. Although negative symptoms were related to more severe schizophrenia, we were unable to demonstrate any impact of other variables on the differences between schizophrenia and schizoaffective disorder/affective psychosis. However, because of the limited nature of the data, these results should be accepted only as preliminary. Third, one of the other potential confounders for the current study may be medication effects. We again tried to 
work out the impact of medication effects on cognition by metaregression methods and did not demonstrate any effect of between-group differences of antipsychotic doses (chlorpromazine equivalents) on cognition. However, since only 10 studies reported such data, we were unable to exclude the potential of medication to explain some of the observed group differences. Future studies should investigate cognitive functions in relation to subtypes of schizophrenia and mood disorders and studies need to more carefully match for confounder variables.

Emre Bora, MD, Melbourne Neuropsychiatry Centre, National Neuroscience Facility, Carlton South and Melbourne Health, Victoria; Murat Yucel, PhD, Melbourne Neuropsychiatry Centre, National Neuroscience Facility, Carlton South, Melbourne Health, Victoria and ORYGEN Research Centre, Parkville, Victoria, Australia;

Christos Pantelis, MD, Melbourne Neuropsychiatry Centre, National Neuroscience Facility, Carlton South and Melbourne Health, Victoria, Australia

Correspondence: Emre Bora, Melbourne Neuropsychiatry Centre, Alan Gilber Building, National Neuroscience Facility, Level 3, Victoria 3053, Australia. Email: emrebora@hotmail.com

First received 12 Jun 2008, final revision 2 Mar 2009, accepted 9 Jun 2009

\section{References}

1 Owen MJ, Craddock N, Jablensky A. The genetic deconstruction of psychosis Schizophr Bull 2007; 33: 905-11.

2 Angst J. Psychiatric diagnoses: the weak component of modern research. World Psychiatry 2007; 6: 30-1.

3 Craddock N, Owen MJ. Rethinking psychosis: the disadvantages of a dichotomous classification now outweight the advantages. World Psychiatry 2007; 6: 84-91.

4 Bora E, Yucel M, Fornito A, Berk M, Pantelis C. Major psychoses with mixed psychotic and mood symptoms: are mixed psychoses are associated with different neurobiological markers? Acta Psychiatr Scand 2008; 118: 172-87.

5 Robinson $\sqcup$, Thompson JM, Gallagher P, Goswami U, Young AH, Ferrier IN, et al. A meta-analysis of cognitive deficits in euthymic patients with bipolar disorder. J Affect Disord 2006; 93: 105-15.

6 Torres IJ, Boudreau VG, Yatham LN. Neuropsychological functioning in euthymic bipolar disorder: a meta-analysis. Acta Psychiatr Scand Suppl 2007; 434: $17-26$.

7 Bora $E$, Yucel $M$, Pantelis C. Cognitive endophenotpes of bipolar disorder: A meta-analysis of neuropsychological deficits in euthymic patients and their first-degree relatives. J Affect Disord 2009; 113: 1-20.

8 Krabbendam L, Arts B, Van Os J, Aleman A. Cognitive functioning in patients with schizophrenia and bipolar disorder: a quantitative review. Schizophr Res 2005; 80: 137-49.

9 Pantelis C, Stuart GW, Nelson HE, Robbins TW, Barnes TRE. Spatial working memory deficits in schizophrenia: relationship with tardive dyskinesia and negative symptoms. Am J Psychiatry 2001; 158: 1276-85

10 Gruzelier J, Seymour K, Wilson L, Jolley A, Hirsch S. Impairments on neuropsychological tests of temporohippocampal and frontohippocampal functions and word fluency in remitting schizophrenia and affective disorders. Arch Gen Psychiatry 1988; 45: 623-9.

11 Bornstein RA, Nasrallah HA, Olson SC, Coffman JA, Torello M, Schwarzkopf SB. Neuropsychological deficit in schizophrenic subtypes: paranoid, nonparanoid, and schizoaffective subgroups. Psychiatry Res 1990; 31: 15-24.

12 Morice R. Cognitive inflexibility and pre-frontal dysfunction in schizophrenia and mania. Br J Psychiatry 1990; 157: 50-4.

13 Beatty WW, Jocic Z, Monson N, Staton RD. Memory and frontal lobe dysfunction in schizophrenia and schizoaffective disorder. J Nerv Ment Dis 1993; 181: 448-53.

14 Jeste DV, Heaton SC, Paulsen SJ, Ercoli L, Harris MJ, Heaton RK. Clinical and neuropsychological comparison of psychotic depression with nonpsychotic depression and schizophrenia. Am J Psychiatry 1996; 153: 490-6.

15 Miller LS, Swanson-Green T, Moses JA, Faustman WO. Comparison of cognitive performance in RDC diagnosed schizoaffective and schizophrenic patients with the Luria-Nebraska neuropsychological battery. J Psychiatr Res 1996; 30: 277-82.

16 Manschreck TC, Maher BA, Beaudette SM, Redmond DA. Context memory in schizoaffective and schizophrenic disorders. Schizophr Res 1997; 26: 153-61.
17 Evans JD, Heaton RK, Paulsen JS, McAdams LA, Heaton SC, Jeste DV. Schizoaffective disorder: a form of schizophrenia or affective disorder? J Clin Psychiatry 1999; 60: 874-82.

18 Mojtabai R, Bromet EJ, Harvey PD, Carlson GA, Craig TJ, Fennig S. Neuropsychological differences between first-admission schizophrenia and psychotic affective disorders. Am J Psychiatry 2000; 157: 1453-60.

19 Rossi A, Arduini L, Daneluzzo E, Bustini M, Prosperini P, Stratta P. J Psychiatr Res 2000; 34: 333-9.

20 Verdoux $\mathrm{H}$, Liraud F. Neuropsychological function in subjects with psychotic and affective disorders. Relationship to diagnostic category and duration of illness. Eur Psychiatry 2000; 15: 236-43.

21 Gilvarry CM, Russell A, Jones P, Sham P. Hemsley P, Murray RM. Verbal fluency in patients with schizophrenia and affective psychoses and their first degree relatives. Psychol Med 2001; 31: 695-704.

22 Gooding DC, Tallent KA. Spatial working memory performance in patients with schizoaffective psychosis versus schizophrenia: a tale of two disorders? Schizophr Res 2002; 53: 209-18.

23 Seidman LJ, Kremen WS, Koren D, Faraone SV, Goldstein JM, Tsuang MT. A comparative profike analysis of neuropsychological functioning in patients with schizophrenia and bipolar psychoses. Schizophr Res 2002; 53: 31-44.

24 Arduini L, Kalyvoka A, Stratta P, Rinaldi O, Daneluzzo E, Rossi A. Insight and neuropsychological function in patients with schizophrenia and bipolar disorder with psychotic features. Can J Psychiatry 2003; 48: 338-41.

25 Fitzgerald D, Lucas S, Redoblado MA, Winter V, Brennan J, Anderson J, et al. Cognitive functioning in young people with first-episode psychosis: relationship to diagnosis and clinical characteristics. Aus N Z J Psychiatry 2004; 38: 501-10.

26 Wiegand LC, Warfield SK, Levitt JJ, Hirayasu Y, Salisbury DF, Heckers S, et al. Prefrontal cortical thickness in first-episode psychosis: a magnetic resonance imaging study. Biol Psychiatry 2004; 5: 131-40.

27 Badcock JC, Michie PT, Rock D. Spatial working memory and planning ability: contrasts between schizophrenia and bipolar I disorder. Cortex 2005; 41: 753-63.

28 Goldstein G, Shemansky WJ, Allen DN. Cognitive function in schizoaffective disorder and clinical subtypes of schizophrenia. Arch Clin Neuropsychol 2005: 20; 153-9.

29 Stip E, Sepehry AA, Prouteau A, Briand C, Nicole L, Lalolende P, et al. Cognitive discernible factors between schizophrenia and schizoaffective disorder. Brain Cogn 2005; 59: 292-5.

30 Glahn DC, Bearden CE, Cakir S, Barrett JA, Najt P, Monkul ES, et al. Differential working memory impairment in bipolar disorder and schizophrenia: effects of life time history of psychosis. Bipolar Disord 2006; 8: 117-23.

31 Gruber O, Gruber E, Falkai P. Articulatory rehearsal in verbal working memory: a possible neurocognitive endophenotype that differentiates between schizophrenia and schizoaffective disorder. Neurosci Let 2006; 405 24-8.

32 Fiszdon JM, Richardson R, Greig T, Bell MD. A comparison of basic and social cognition between schizophrenia and schizoaffective disorder. Schizophr Res 2007; 91: 117-21.

33 Selva G, Salazar J, Balanza-Martinez V, Martinez-Aran A, Rubio C, Daban C, et al. Bipolar patients with and without a history of psychotic symptoms: do they differ in cognitive functioning? J Psychiatr Res 2007; 41: 265-72.

34 Szoke A, Meary A, Trandafir A, Bellivier F, Roy I, Schurhoff F, et al. Executive deficits in psychotic and bipolar disorders. Implications for our understanding of schizoaffective disorder. Eur Psychiatry 2008; 23: 20-5.

35 Heinrichs RW, Ammari N, Vaz SM, Miles AA. Are schizophrenia and schizoaffective disorder are distinguishable? Schizophr Res 2008; 99: 149-54.

36 Ayres MA, Busatto GF, Menezes PR, Schaufelberger MS, Coutinho L, Murray RM, et al. Cognitive deficits in first episode psychosis: a population based study in Sao Paulo, Brazil. Schizophr Res 2007; 90: 338-43.

37 Gilvarry CM, Barber JS, Van Os J, Murray RM. Neuropsychological performance of psychotic patients in community care: results from the UK700 study. Acta Psychiatr Scand Suppl 2001; 104: 81-91.

38 Metz JT, Johnson MD, Pliskin NH, Luchins DJ. Maintenance of training effects of the Wisconsin Card Sorting Test by patients with schizophrenia or affective disorders. Am J Psychiatry 1994; 151: 120-2.

39 Savage RM, Jackson WT, Sourathathone CM. A brief neuropsychological testing battery for evaluating patients with schizophrenia. Community Ment Health J 2003; 39: 253-62.

40 Taiminen $\mathrm{T}$, Jaaskelainen $\mathrm{S}$, Ilonen $\mathrm{T}$, Meyer $\mathrm{H}$, Karlsson $\mathrm{H}$, Lauerma $\mathrm{H}$, et al. Habituation of the blink reflex in first-episode schizophrenia, psychotic depression and non-psychotic depression. Schizophr Res 2000; 44: 69-79.

41 Reitan RM. Validity of Trail Making Test as an indication of organic brain disease. Percept Mot Skills 1958; 8: 271-6. 
42 Wechsler D. WAIS-III. Wechsler Adult Intelligence Scale, Administration and Scoring Manual (3rd edn). Psychological Corporation, 1997.

43 Heaton RK. Wisconsin Card Sorting Test Manual. Psychological Assessment Resources, 1981.

44 Lezak MD, Howieson, DB, Loring DW. Neuropsychological Assessment, (4th edn). Oxford University Press, 2004.

45 Gold JM, Carpenter C, Randolph C, Goldberg TE, Weinberger DR. Auditory working memory and Wisconsin Card Sorting Test performance in schizophrenia. Arch Gen Psychiatry 1997; 54: 159-65.

46 Robbins TW, James M, Owen AM, Sahakian BJ, Mclnnes L, Rabbitt P. Cambridge Neuropsychological Test Automated Battery (CANTAB): a factor analytical study of large sample of normal elderly volunteers. Dementia 1994 5: $266-81$.

47 Rey A. L'examen Clinique en Psychologie. Presses Universitaires de France, 1964.

48 Delis DC, Kramer JH, Kaplan E, Ober BA. California Verbal Learning Test: Adult Version. Psychological Corporation, 1987.

49 Signoret JL. BEM-84, Batterie d'Efficience Mnesique Reduite (Abbreviated battery of memory efficiency). Editions Elsevier, 1991.

50 Brandt J, Benedict RHB. The Hopkins Verbal Learning Test-Revised: Professional Manual. Psychological Assessment Resources, 2001.

51 Miller G, Selfridge J. Verbal context and recall of meaningful material. Am J Psychol 1950; 63: 176-85.

52 Wechsler D. Wechsler Memory Scale - Revised Manual. Psychological Corporation, 1987.

53 Rey A. Psychological examination of traumatic encephalopathy. Archives de Psychologie 1941; 28: 286-340.

54 Benton A. Benton Visual Retention Test, (4th edn). Psychological Corporation, 1974.

55 Van Beilen M, Pijnenborg M, van Zomeren EH, van den Bosch RJ, Withaar FK, Bouma A. What is measured by verbal fluency tests in schizophrenia? Schizophr Res 2004; 69: 267-76.

56 Bax L, Yu LM, Ikeda N, Tsuruta H, Moons KGM. Development and validation of MIX: comprehensive free software for meta-analysis of causal research data. BMC Med Res Methodol 2006; 6: 50.
57 Leung A, Chue P. Sex differences in schizophrenia, a review of the literature. Acta Psychiatr Scand Suppl 2000; 401: 3-38.

58 Roy MA, Maziade M, Labbe A, Merette C. Male gender is associated with deficit schizophrenia: a meta-analysis. Schizophr Res 2001; 47: 141-7.

59 Cohen AS, Saperstein AM, Gold JM, Kirkpatrick B, Carpenter Jr WT, Buchanan RW. Neuropsychology of the deficit syndrome: new data and meta-analysis of findings to date. Schizophr Bull 2007; 33: 1201-12.

60 Kempf L, Hussain N, Potash JB. Mood disorders with psychotic featurs, schizoaffective disorder, and schizophrenia with mood features: trouble at the borders. Int Rev Psychiatry 2005; 17: 9-19.

61 Kirckpatrick B, Buchanan RW, Breier A, Carpenter WT. Depressive symptoms and the deficit syndrome of schizophrenia. J Nerv Ment Dis 1994; 182: 452-5.

62 Fleming SK, Blasey C, Schatzberg AF. Neuropsychological correlates of psychotic features in major depressive disorders: a review and metaanalysis. J Psychiatr Res 2004: 38: 27-35.

63 Bora E, Vahip S, Akdeniz F, Gonul AS, Eryavuz A, Ogut M, et al. The effect of previous psychotic mood episodes on cognitive impairment in euthymic bipolar patients. Bipolar Disord 2007: 9: 468-77.

64 Glahn DC, Bearden CE, Barguil M, Barrett J, Reichenberg A, Bowden CL, et al. The neurocognitive signature of psychotic bipolar disorder. Biol Psychiatry 2007; 62: 910-6.

65 Crow T. Nature of the genetic contribution to psychotic illness - a continuum view point. Acta Psychiatr Scand 1990; 81: 401-8.

66 Lake CR, Hurwitz N. Schizoaffective disorder merges schizophrenia and bipolar disorders as one disease - there is no schizoaffective disorder. Curr Opin Psychiatry 2007; 20: 365-79.

67 Hallmayer JF, Kalaydjeiva L, Badcock J, Dragovic M, Howell S, Michie PT, et al. Genetic evidence for distinct subtype of schizophrenia characterized by pervasive cognitive deficit. Am J Hum Genet 2005; 77: 468-76.

68 Keefe RSE, Fenton WS. How should DSM-V criteria for schizophrenia include cognitive impairment? Schizophr Bull 2007; 33: 912-20. 